Document downloaded from:

http://hdl.handle.net/10251/176370

This paper must be cited as:

Chillarón-Pérez, M.; Vidal-Gimeno, V.; Verdú Martín, GJ. (2020). CT image reconstruction with SuiteSparseQR factorization package. Radiation Physics and Chemistry. 167:1-7. https://doi.org/10.1016/j.radphyschem.2019.04.039

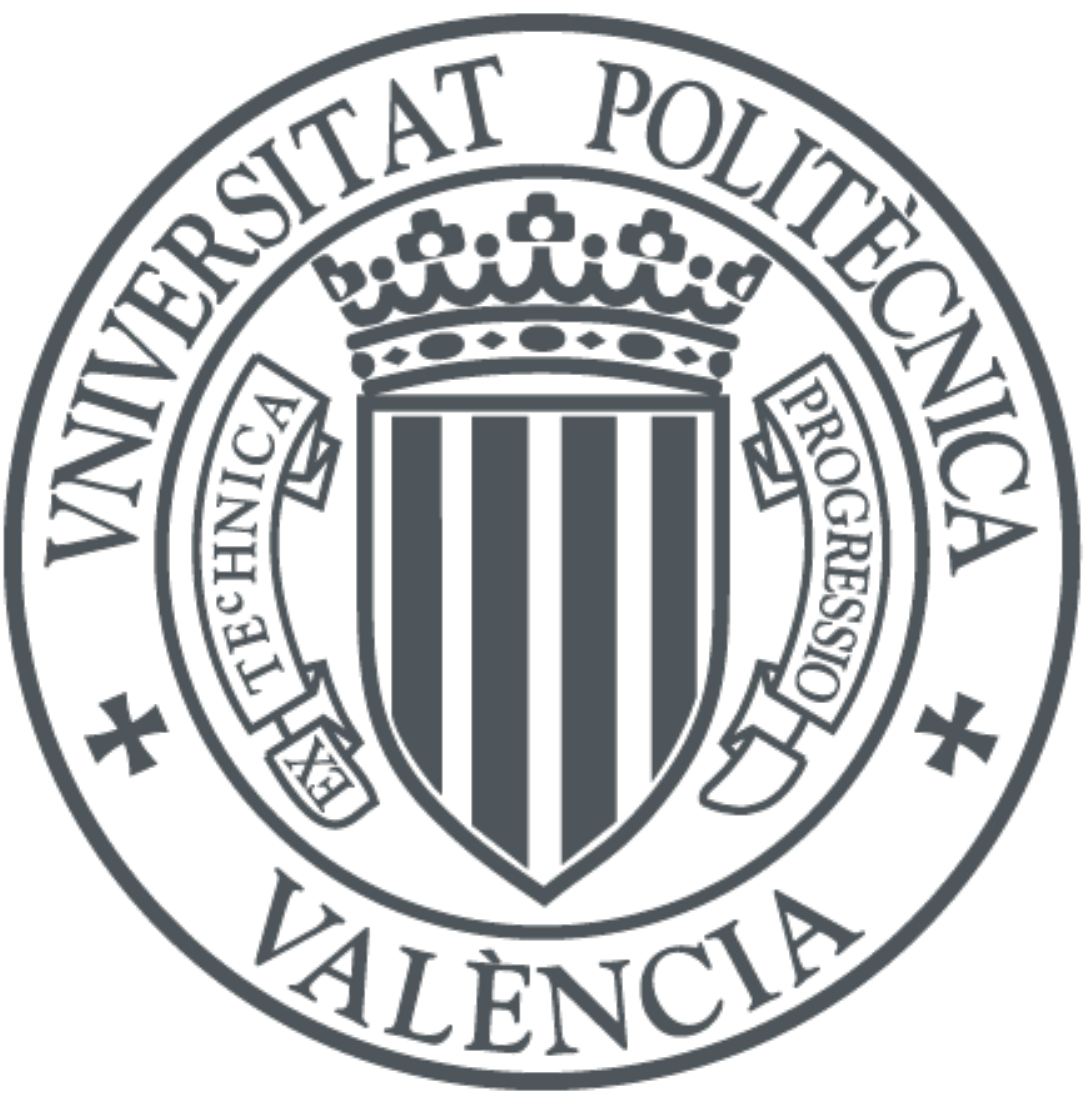

The final publication is available at

https://doi.org/10.1016/j.radphyschem.2019.04.039

Copyright Elsevier

Additional Information 


\title{
CT image reconstruction with SuiteSparseQR factorization package
}

\author{
Mónica Chillarón ${ }^{\mathrm{a}, *}$, Vicente Vidal ${ }^{\mathrm{a}}$, Gumersindo Verdú ${ }^{\mathrm{b}}$ \\ ${ }^{a}$ Departamento de Sistemas Informáticos y Computación (DSIC). Universitat Politècnica de València. Camí de Vera S/n. \\ València. 46022 Spain. \\ ${ }^{b}$ Instituto de Seguridad Industrial, Radiofísica y Medioambiental (ISIRYM). Universitat Politècnica de València. Camí de \\ Vera $S / n$. València. 46022 Spain.
}

\begin{abstract}
SuiteSparseQR is a factorization package for sparse matrices oriented to parallelism in multicore architectures. It employs BLAS and LAPACK as well as Intel's Threading Building Blocks to achieve high performance. Through the SPQR method implemented in this package we can use the QR decomposition to reconstruct $\mathrm{CT}$ images efficiently. In this paper, we analyze the behavior of the package applied to the reconstruction of medical CT images, studying the quality of the obtained image. To this purpose, we use the image dataset DeepLesion, which provides various CT studies of different lesions in different organs or tissues. We also compare it to our previous iterative reconstruction method called LSQR. This new method is promising since the computations are simplified if we compare it to the iterative options and the reconstructions are high-quality, as the results show.
\end{abstract}

Keywords: SuiteSparse, QR, SPQR, LSQR, CT, Reconstruction, Medical Imaging, DeepLesion

\section{Introduction}

Currently, Computerized Tomography (CT) studies are well established in clinical practice for the diagnosis of multiple diseases as well as for their monitorization. Unlike other medical imaging methods for diagnostics such as Magnetic Resonance Imaging (MRI) or ultrasounds, the high power X-rays generated 5 during CT scanning is a hazard for all patients, especially for vulnerable patients such as children or pregnant women, and also cancer patients who require regular scans to assess the evolution of the disease (Kak and Slaney, 2001).

Several studies have shown the generalized increase of cancer incidence all over the world, and also the projection of cancer-provoked deaths by 2020 and 2030 (Rahib et al., 2014). For instance, the risk of having any type of cancer is about a 30\% nowadays in the European Union, according to the The International Agency for Research on Cancer ("IARC"). The statistics for 2018 are summarized in (Bray et al., 2018). Also the projections show an decrease of mortality rates but the incidence is going up in general terms, and at a higher pace for several types such as lung, breast or thyroid cancer.

Since the radiation produced by an X-ray machine can induce cancer by itself, it is necessary to develop new methods that can allow reducing it to the maximum while still having a good image quality that helps physicians make accurate diagnostics.

Since the issue of radiation came across, the reconstruction techniques for CT scans have significantly changed. When this clinical test started, they used methods that were based on the Fourier transform. They are called analytical reconstruction methods and to this day they remain being the most used in clinical practice, being all modifications of the filtered-backprojection (FBP) algorithm (Feldkamp et al., 1984; Tang et al., 2006). That is due to their fast reconstructing times, since you can have a complete CT scan in a matter of seconds.

\footnotetext{
* Corresponding author

Email addresses: mnichipr@inf.upv.es (Mónica Chillarón), vvidal@dsic.upv.es (Vicente Vidal), gverdu@iqn.upv.es (Gumersindo Verdú)
} 
However, they are not the best option regarding radiation, since they need a complete set of projections to obtain a good-quality image. If the number of projections is reduced, the resulting images have artifacts such as rings or beam-hardening that can mean they are invalid for diagnosis.

On the other hand we find algebraic methods, that can be either iterative or direct. Iterative techniques such as ART (Andersen, 1989), SART (Andersen and Kak, 1984) or LSQR (Flores et al., 2014, 2015; Parcero et al., 2017) perform an approximation to the solution image and they obtain a good balance between radiation dose and image quality since they allow us reducing the numbers of projections taken

so when doing a CT scan. Nevertheless, they are really slow methods since they iterate to approximate the result image.

Direct methods are not widely explored for this field due to their high computational cost for the large matrices we are working with. But with hardware evolving fast and becoming more affordable, It is now possible to compute factorizations of extremely large matrices. If we also take advantage of parallel computing or High-Performance Computing (HPC) it is possible to take the direct algebraic approach to the CT image reconstruction problem.

In this paper we focus on the application of the QR matrix factorization for CT image reconstruction. We perform the QR decomposition in a cluster using the software included in the package SuiteSparseQR (Davis, 2011). This package gives us a lot of basic sparse matrices algebraic algorithms and also provides the possibility of using BLAS threads as well as Intel Threading Building Block (TBB) that can parallelize the factorization code and allows us to exploit our hardware resources to the maximum. In works like (Rodríguez-Alvarez et al., 2018) they perform the QR factorization to reconstruct small CT images but as they mention, the method is not parelellized or optimized to reduce the fill-in of the matrix, which the implementation in this packages does. In our previous work (Chillarón et al., 2018), we did a preliminary study in which we applied the multifrontal sparse QR (SPQR) method to reconstruct phantom images, in order to determine its feasibility. We concluded the maximum resolution we could reach with the hardware we have available is $256 \times 256$ pixels. Besides, we observed we need a full-rank system matrix to reconstruct the images, which in this case is obtained with 90 projection angles.

In Section 2, we will describe the method used to simulate a CT scanner, as well as the way to perform the reconstruction of a CT sinogram using the QR factorization or the LSQR method. We also make a brief description of the images dataset DeepLesion, used to take as reference, and the metrics we use to measure the image quality. In Section 3 we will analize the performance in terms of quality of the CT reconstructions using the QR decomposition. Moreover, the resulting images will be compared to the best quality reconstructions obtained through LSQR so we can determine which method is a better alternative and discuss the disadvantages of each of them. To conclude, in Section 4 we summarize and discuss the advantages of the studied methods and propose a future line of work.

\section{Material and Methods}

\subsection{Computerized Tomography}

The CT image reconstruction problem can be modelled as described in Eq. (1), so it can be be solved by algebraic methods. In this linear equations system, $A$ (Eq. (4)) is the system matrix or weights matrix, which measures the contribution of each ray beam traced $(i)$ to each image pixel $(j)$. We calculate this matrix using the Joseph method (Joseph, 1982). The size of $A$ is $M \times N$, being $M$ the size of the x-ray projections $\left(\mathrm{n}^{\circ}\right.$ of views ${ }^{*} \mathrm{n}^{\circ}$ of detectors) and $N$ the resolution of the image to be reconstructed $(32 \times 32$, $\ldots, 512 \times 512$ pixels). The projection data is $g$ (Eq. (2)), which is the X-ray sinogram represented in vector form. Last we have the vector $u$ (Eq. (3)), or the solution image we get when we solve the system (Eq. (1)) for $u$.

$$
\begin{gathered}
A * u=g \\
g=\left[g_{1}, g_{2}, \ldots, g_{M}\right]^{T} \in \mathbb{R}^{M}
\end{gathered}
$$

$$
\begin{gathered}
u=\left[u_{1}, u_{2}, \ldots, u_{N}\right]^{T} \in \mathbb{R}^{N} \\
A=a_{i, j} \in \mathbb{R}^{M x N}
\end{gathered}
$$




\section{2. $Q R$ reconstruction method}

If the rank of the system matrix $A$ is complete, the problem could be solved directly using its inverse 70 as Eq. (5). But our matrices are too large to explicitly calculate the inverse. It would imply a high computational cost and the accumulating errors could distort the solution image.

Instead, we use the QR factorization (Golub and Ortega, 1993) of $A$ (Eq. (7)), where the matrix $Q$ is orthogonal and $R$ is upper triangular. Besides, we perform the decomposition version with pivoting, where the permutation matrix $P$ is used to reduce the fill-in of the matrices in the factorization process. With this

75 factorization we simulate the inverse as Eq. (8) (or pseudoinverse as Eq. (6) if $A$ is rank-deficient).

In this way we can solve the problem with direct algebraic methods as Eq. (9) and most of the computations can be made in advance and store for later use to reconstruct the image.

$$
\begin{gathered}
u=A^{-1} * g \\
u=A^{+} * g \\
A * P=Q * R
\end{gathered}
$$

$$
\begin{gathered}
A^{-1}=P R^{-1} Q^{T} \\
u=P *\left(R^{-1}\left(Q^{T} * g\right)\right)
\end{gathered}
$$

\subsection{Q-less reconstruction method}

An alternative to the traditional QR factorization is using Householder reflections. This means we don't need to explicitly calculate and store Q. Instead, we can store only the transforms to be applied in Householder form as Eq. (10), where $H_{i}$ are the successive reflection matrices. By doing this, we significantly reduce the computational cost and we spare main memory resources, which is vital to this scenario.

$$
Q=H_{1} H_{2} \cdots H_{m-2} H_{m-1}
$$

\subsection{Least Squares $Q R$ reconstruction method}

The problem in Eq. (1) can also be solved by iterative algebraic methods. The Least Squares QR method, presented in (Paige and Saunders, 1982), is a good choice for sparse and possibly rank-deficient systems as is our case. We verified its validity in our previous works (Flores et al., 2014, 2015; Parcero et al., 2017; Chillarón et al., 2017).

This method is based on the bidiagonalization method by Golub and Kahan (Golub and Kahan, 1965). It solves the system by minimizing $\|g-A u\|_{2}$, generating a sequence of approximations $u_{k}$ such as the 2 -norm of the residue (being the residue $r_{k}=g-A u_{k}$ for iterain Algorithm 1, where $\alpha_{\mathrm{i}}$ and $\beta_{1}$ are chosen to normalize the corresponding vectors. For instance, the operation $\alpha_{1} v_{1}=A^{\mathrm{T}} x_{1}$ implies computing $\overline{v_{1}}=A^{\mathrm{T}} x_{1}, \alpha_{1}=\left\|\overline{v_{1}}\right\|$ and $v_{1}=\left(1 / \alpha_{1}\right) \overline{v_{1}}$.

In our reconstruction method, we combine this iterative method with a regularization technique called Soft Thresholding Filter (STF)(Yu and Wang, 2010; Yu and Zeng, 2014) and an acceleration step called Fast Iterative Shrinkage-Thresholding Algorithm (FISTA) (Beck and Teboulle, 2009). Both of these techniques help with the convergence rate and they are especially needed for ill-conditioned prob-

1. Initialization: $u_{0}=0$

2. Iterate:

- Update the current reconstruction using a fixed number of LSQR iterations.

- Perform the filtering step using STF

- Apply acceleration technique FISTA

- Return to step (2) until the stopping criterion is satisfied or we reach a maximum number of iterations

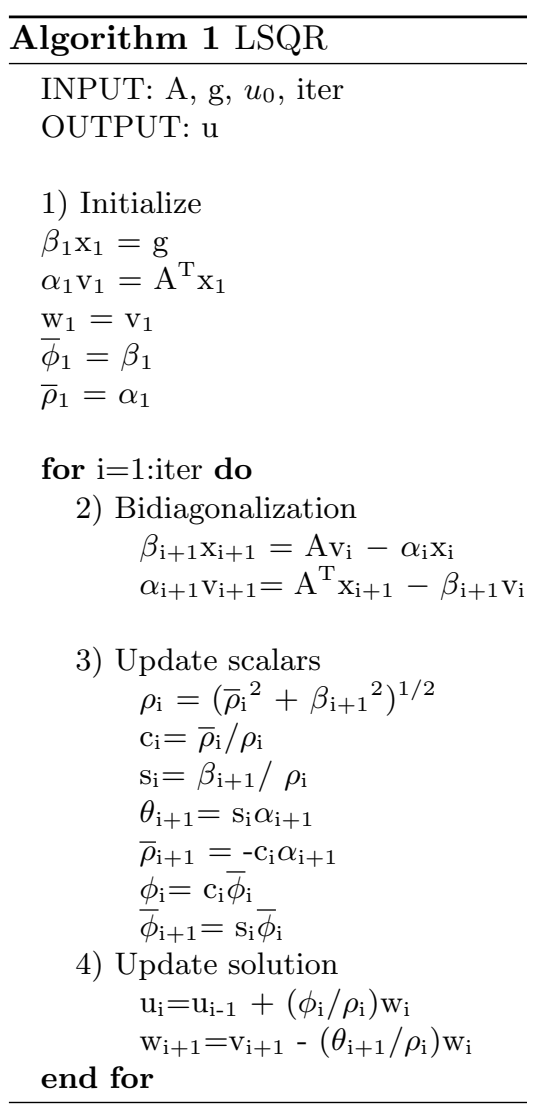


As stopping criterion, we take the relative residual $\|g-A u\|_{2} /\|g\|_{2}$, set to a minimum tolerance which is usually 1e-06. On each iteration, we take the solution modified by FISTA as the initial solution for the next LSQR iteration.

\subsection{Image Quality Metrics}

To measure the quality of the reconstructed images, we have used the metrics PSNR (Peak Signal-ToNoise Ratio) and SSIM (Structural Similarity Index) (Hore and Ziou, 2010). The PSNR metric measures the ratio between the image signal and the noise it contains. To calculate it, another metric is used, the socalled Mean Square Error (MSE), which is calculated according to Eq. (11), and represents the mean of the squared error between the reference image $I_{0}$ and the reconstructed image $u$. Once the MSE is calculated, it is used to calculate the PSNR according to Eq. (12), in which MAX represents the maximum value that a pixel can take. The higher the PSNR value we get, the better the reconstruction we have.

With SSIM we can measure the internal structures (shapes) of the images compared with the reference image. Therefore, it does not look at the gray levels of the pixels, but the shapes of the reconstructed image with respect to the reference image, and therefore measures what is perceptible to the human eye. It is applied through windows of fixed size, and the difference between two windows $x$ and $y$ corresponding to the two images to be compared is calculated, using Eq. (13). In this equation, $\mu_{x}$ and $\mu_{y}$ denote the average value of the window $x$ and $y, \sigma_{x}^{2}$ and $\sigma_{y}^{2}$ the variance, $\sigma_{x y}$ the covariance between the windows, and $c_{1}$ and $\mathrm{c}_{2}$ are two stabilizing variables dependent on the dynamic range of the image.

$$
\begin{gathered}
M S E=\frac{1}{M N} \sum_{i=0}^{M-1} \sum_{j=0}^{N-1}\left\|I_{0}(i, j)-u(i, j)\right\|^{2} \\
P S N R=10 * \log _{10} \frac{M A X_{I_{0}}^{2}}{M S E} \\
S S I M=\frac{\left(2 \mu_{x} \mu_{y}+c_{1}\right)\left(2 \sigma_{x, y}+c_{2}\right)}{\left(\mu_{x}^{2}+\mu_{y}^{2}+c_{1}\right)\left(\sigma_{x}^{2}+\sigma_{y}^{2}+c_{2}\right)}
\end{gathered}
$$

\subsection{CT Images Dataset}

To test the validity of our reconstructions, we used images pertaining to DeepLesion (Yan et al., 2018), a dataset with 32,735 lesions in 32,120 CT slices from 10,594 studies of 4,427 unique patients. In this images collection we can find CT studies for different types of lesions, with tags that divide them into eight classes: Bone, Abdomen (that are not Liver or Kidney), Mediastinum, Liver, Lung, Kidney, Soft tissue and Pelvis.

We choose one image for each lesion category, shown in Fig. 1. The images were chosen randomly, one for each type of lesion, and selected from a list of the key slices from each study provided by the authors. Since these images are already reconstructions, it is implied the scanner software used its own algorithms to try to remove Poisson noise, as well as the artifacts due to detectors noise or beam hardening. But the images we use as reference can still have some noise since they are real reconstructions.

This dataset gives us the required variety of images to test our reconstructions, both with direct and iterative methods. For this purpose, we project the selected images with Joseph (Joseph, 1982) method as we described in Section 2.1. Although the original resolution of the images is $512 \times 512$ pixels, we resize them to $256 \times 256$ pixels since it's the highest resolution we can compute at the moment. The sinograms were simulated for a scanner with 1025 detectors and using 90 views along the 360 degrees of rotation. Then, we measure the quality of the results with the metrics PSNR and SSIM (Hore and Ziou, 2010) and we also try 


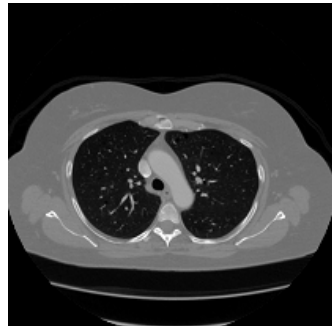

(a) Bone

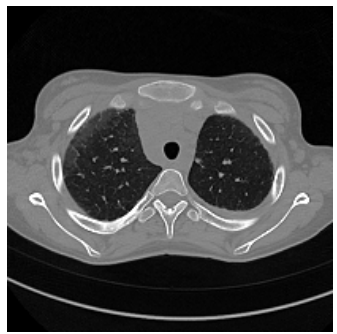

(e) Lung

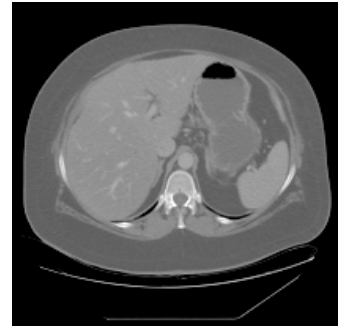

(b) Abdomen

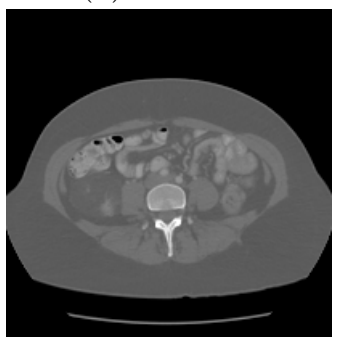

(f) Kidney



(c) Mediastinum

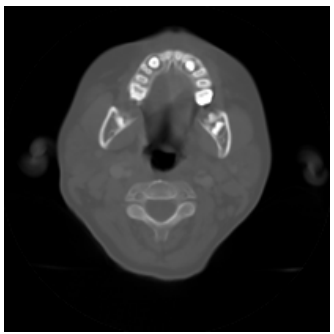

(g) Soft Tissue

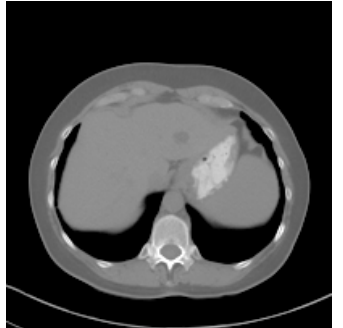

(d) Liver

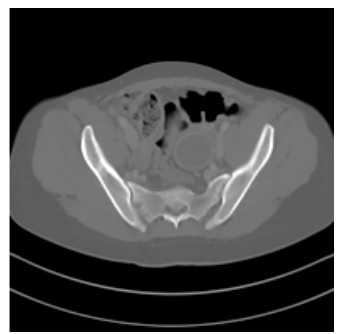

(h) Pelvis

Figure 1: Selected images classified by type of lesion

\section{Results and Discussion}

We have used the eight images of selected lesions to reconstruct with each method and compare the results obtained. All the computations have been made in an HPC cluster with four Intel Xeon E5-4620 8c/16T processors (8 cores/processor, 32 cores/node) and 256GB DDR3 RAM memory (ratio 8GB/core), using up to 32 threads.

With SPQR, the reconstruction is trivial since it consists of computing Eq. (9), using the QR factorization previously performed and stored. With LSQR, the process is more complex, since as we analyzed in our previous work (Chillarón et al., 2017), in our method (which combines LSQR, the STF filter, and FISTA acceleration) several parameters need to be adjusted in order to get the better result possible. Therefore, the reconstruction has to be supervised since there is no guarantee of convergence.

In Fig. 2 we show the PSNR (Peak Signal-to-Noise Ratio) and SSIM (Structural Similarity Index) of the two techniques for each lesion image. To measure this, we selected the image region that has the body part, eliminating the outer region with only air.

As we can see in Fig. 2a, the reconstructions obtained by LSQR seem to be of much lower quality than those obtained by QR reconstruction, having a difference of around 200 in the PSNR value. The reconstructions with the $\mathrm{QR}$ can be considered noiseless with these results, compared to the reference image. The mean squared error (MSE) is of the order of 1e-24 in this case, while for the reconstructions with LSQR it is of the order of 1e-05. Therefore, reconstructions with the iterative method have more noise, while the ones obtained with the QR are practically identical to the reference image. These results are logical since the iterative method is stopped at a desired tolerance, while the reconstruction with the $\mathrm{QR}$ is direct and the result is not approximated but calculated.

Regarding the SSIM results, shown in Fig. 2b, the difference is not as big. In every case, the QR reconstructions SSIM value is 1, which is the maximum possible. Nevertheless, for the LSQR the minimum SSIM is 0.996 , which can be considered a high-quality result.

For some lesions (Bone, Mediastinum and Soft Tissue) the SSIM value is above 0.999. This means even though the reconstructions could be noisy, the internal structures of the CT images are well preserved generally speaking. But we could still be losing relevant information with LSQR, since SSIM is not always 1, which doesn't happen with the direct method. 


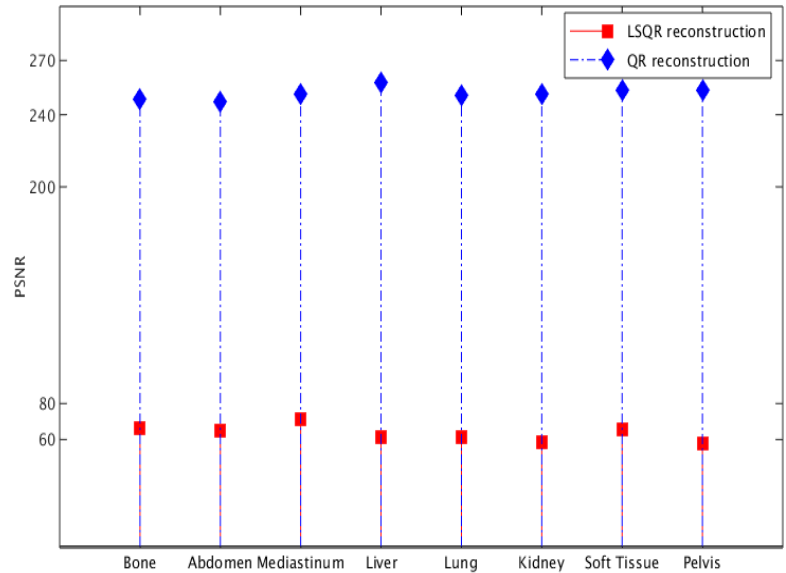

(a) PSNR results

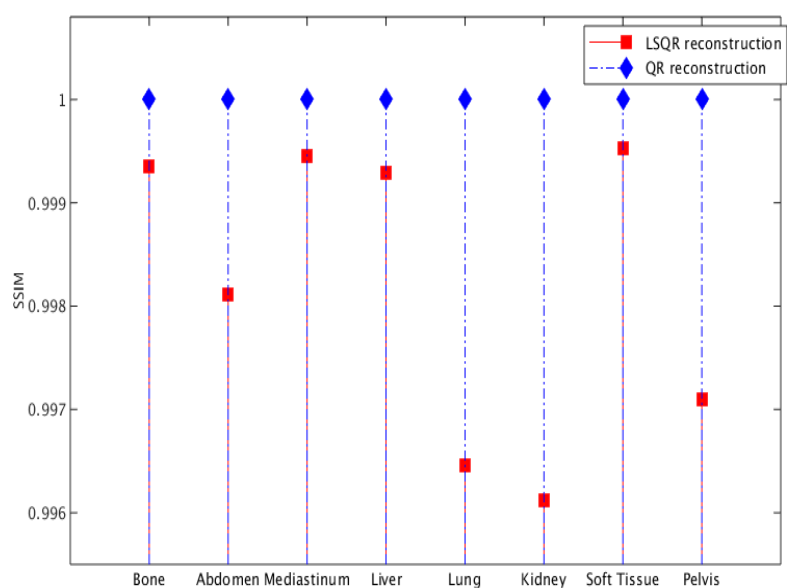

(b) SSIM results

Figure 2: Quality of the reconstructions

To visually check if we lose information, we show the reconstructed images in Fig. 4 to Fig. 11, each one with the lesion's bounding box marked with a green rectangle in the reference image, as well as in the reconstructions. We show the appropriate Hounsfield window to correctly visualize each one of the lesions, as instructed in the dataset information.

In every case, we have been able to locate the lesion in both of the reconstructed images. In addition, we can see the LSQR results are better than expected since the numerical error is not visually discernible when showing the relevant window. So even if the quality metrics indicate a significant difference, we don't perceive it as such. We can notice a slight blurriness in every LSQR reconstruction, but it does not alter the image much.

As an example of this, we provide a zoomed-in image of the reconstructions corresponding to the Liver lesion in Fig. 3. As we can see, the area of the liver is smoother on the LSQR reconstruction, which differs from the reference image (Fig. 7). In addition, the edges are slightly more defined on the QR reconstruction, as can be observed in the lesion bounding box. But the difference is still small, as it is for the other lesion reconstructions.

In some particular cases, as can be the image corresponding to the Mediastinum lesion (Fig. 6), we observe that the QR reconstruction is identical to the reference, but not the LSQR. When windowing the image to the desired Hounsfield Window, we lose some information. That does nor mean the information is not there, just that by windowing we can not see it because the Hounsfield value is not exactly the same in the LSQR reconstruction.

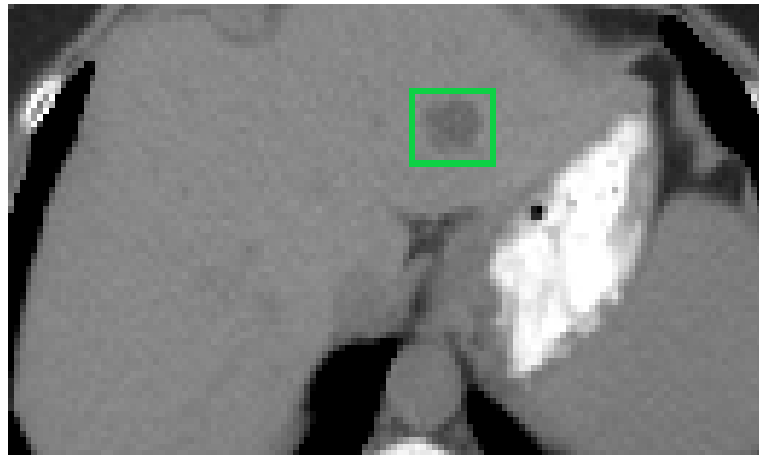

(a) QR rec.

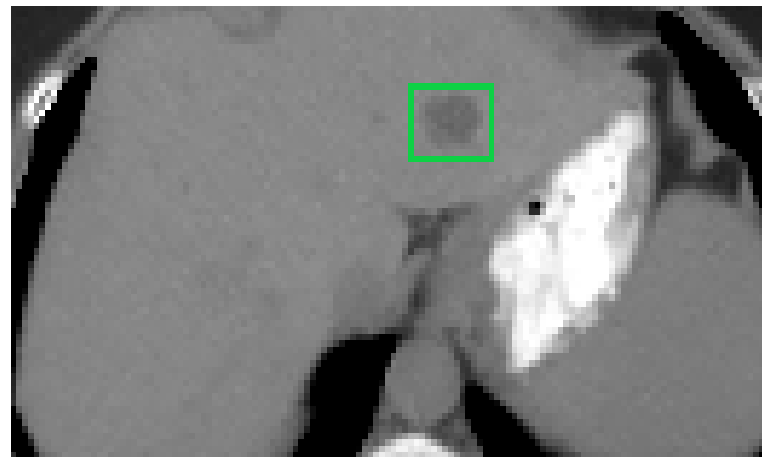

(b) LSQR rec.

Figure 3: Liver Lesion (zoomed-in) 
(a) Reference
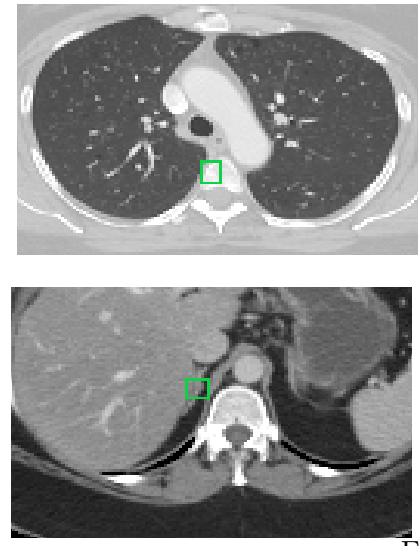

Fig

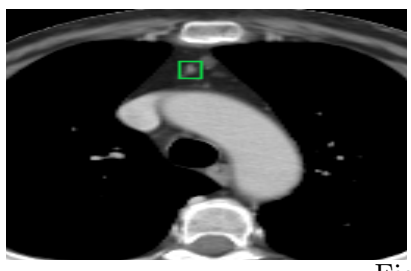

Fig
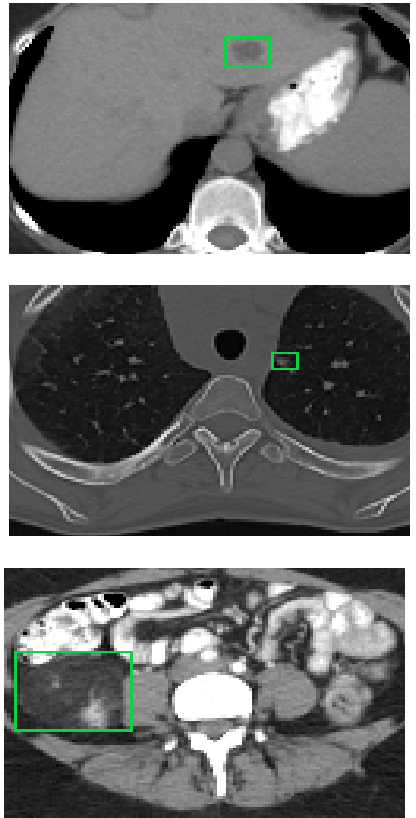

Figure 8: Lung Lesion. HU Window $=[-1$

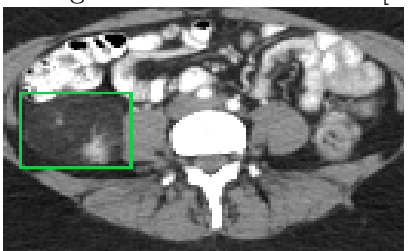

Figure 9: Kidney Lesion. HU Window $=[-160,240$
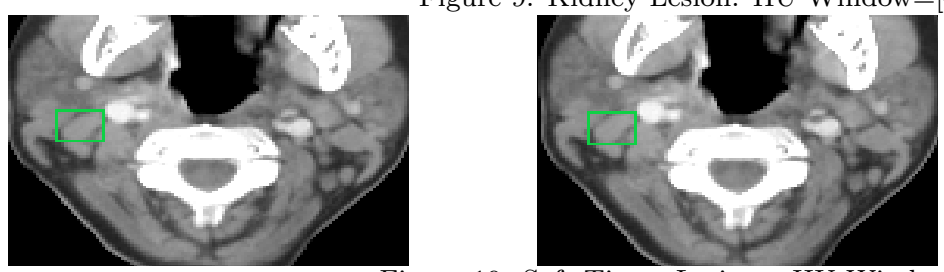

Figure 10: Soft Tissue Lesion. HU Window $=[-160,240]$

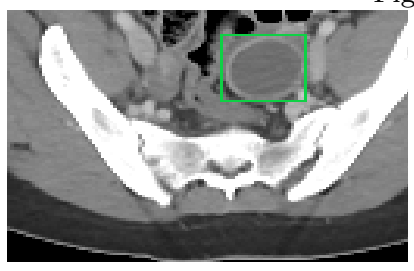

(c) LSQR rec.


Figure 11: Pelvis Lesion. HU Window $=[-175,275]$ 


\section{Conclusion}

In the present work, we have determined the validity of a direct algebraic method for the reconstruction of CT images. To do this, we have used various images belonging to the DeepLesion dataset, representing different parts of the body with different types of lesions.

In our previous work (Chillarón et al., 2018), we determined that the case with the highest resolution that we can solve by means of the QR factorization is for images of 256x256 pixels. This is because we need the system matrix A that models the CT scanner to have full rank to get valid results. Therefore, we have to take at least 90 projections. If we went up to $512 \times 512$ pixels resolution, we would have to use 260 views, and the A matrix would be of size 266500x262144 in our simulations. This case is not computable with the algorithms provided by SuiteSparse and the hardware that we currently have. However, it is possible to solve it by means of LSQR, since we don't factorize the matrix which is always sparse, just operate with it performing matrix-vector products. Therefore it needs less computational resources. In addition, for LSQR we do not need the matrix to be full rank. We can obtain good reconstructions with fewer projections, as shown in our previous works (Chillarón et al., 2017; Parcero et al., 2017). Therefore, although using QR we can reduce the number of projections with respect to analytical methods, we do it to a lesser extent than with LSQR.

On the other hand, the reconstruction is much simpler with the direct method, since we can factorize the matrix once before the reconstruction and have it always stored. When reconstructing, we solve Eq. (9), which is quite fast. In this case, we solved a full volume with 128 slices in less than 30 minutes, depending on the number of cores used, using the Householder form to store Q. If we used the explicit Q, the time required to reconstruct the same volume is doubled. To reduce this time and use less resources, we are working on a new QR factorization algorithm that uses out-of-core techniques to read the data stored in blocks only when it is needed, so we can reconstruct higher resolutions. In addition, since it is a direct operation we can delimit the time it will take depending on the hardware used.

This is not possible with LSQR, since it is an iterative method. We never know how many iterations it will need to converge or if it will converge. We could set it to a number of iterations to delimit the time, but in this way, we can not guarantee a minimum tolerance. In addition, the selection of parameters that influence reconstruction should be supervised. Besides, we currently don't have a tested implementation to solve several right-hand-side vectors with LSQR, so we need to solve each slice independently, unlike with the QR. All in all, we're talking about several hours to get a full volume reconstruction. However, we are working on implementing a Block LSQR using parallel libraries to reconstruct several right-hand-sides, and our preliminary tests show a similar performance than the QR reconstructions using the Householder form when the parameters are well selected.

Regarding the image quality, we have been able to observe that the reconstructions that are obtained applying the direct method based on the QR decomposition are perfect, practically speaking. It can be said that there is no error with respect to the reference images. Nevertheless, it must be noted that in our simulations we are not considering or removing the possible noise generated in the acquisition process of a real CT scanner. In addition, we have compared them to the reconstructions obtained by our previous method based on LSQR. These reconstructions show worse quality from the numerical point of view, which we have verified analyzing the results with both PSNR and SSIM metrics. However, when viewing the images we see that even LSQR obtains reliable results since we are only able to appreciate a slight blurring in some areas.

For all the above we determined that both methods are valid to reconstruct CT images with an algebraic approach. However, the accuracy of the QR method as well as the simplicity of the process is preferable from the point of view of image quality. Nevertheless, this method requires greater computational resources, as well as a greater number of views than the LSQR, with which we can solve larger problems.

\subsection{Acknowledgements}

This research has been supported by "Universitat Politècnica de València", "Generalitat Valenciana" under PROMETEO/2018/035 co-financed by FEDER funds, as well as ACIF/2017/075 predoctoral grant, and the "Spanish Ministry of Economy and Competitiveness" under Grant TIN2015-66972-C5-4-R and TIAMHA co-financed by FEDER funds. 
Andersen, A.H., 1989. Algebraic reconstruction in CT from limited views. IEEE Transactions on Medical Imaging 8, $50-55$. Andersen, A.H., Kak, A.C., 1984. Simultaneous algebraic reconstruction technique (SART): a superior implementation of the ART algorithm. Ultrasonic Imaging 6, 81-94.

Beck, A., Teboulle, M., 2009. A fast iterative shrinkage-thresholding algorithm for linear inverse problems. SIAM Journal on Imaging Sciences 2, 183-202.

Bray, F., Ferlay, J., Soerjomataram, I., Siegel, R.L., Torre, L.A., Jemal, A., 2018. Global cancer statistics 2018: GLOBOCAN estimates of incidence and mortality worldwide for 36 cancers in 185 countries. CA: a cancer journal for clinicians

Chillarón, M., Vidal, V., Segrelles, D., Blanquer, I., Verdú, G., 2017. Combining grid computing and Docker containers for the study and parametrization of CT image reconstruction methods. Procedia Computer Science 108, 1195-1204.

Chillarón, M., Vidal, V., Verdú, G., Arnal, J., 2018. CT Medical Imaging Reconstruction Using Direct Algebraic Methods with Few Projections, in: Computational Science - ICCS 2018, Springer International Publishing, Cham. pp. 334-346.

Davis, T.A., 2011. Algorithm 915, SuitesparseQR: Multifrontal Multithreaded Rank-revealing sparse QR factorization. ACM Trans. Math. Softw. 38, 8:1-8:22.

Feldkamp, L.A., Davis, L., Kress, J.W., 1984. Practical cone-beam algorithm. J Opt Soc Am 1, $612-619$.

Flores, L., Vidal, V., Verdú, G., 2015. Iterative reconstruction from few-view projections. Procedia Computer Science 51, $703-712$.

Flores, L.A., Vidal, V., Mayo, P., Rodenas, F., Verdú, G., 2014. Parallel CT image reconstruction based on GPUs. Radiation Physics and Chemistry 95, 247-250.

Golub, G., Kahan, W., 1965. Calculating the singular values and pseudo-inverse of a matrix. Journal of the Society for Industrial and Applied Mathematics .

Golub, G.H., Ortega, J.M., 1993. Scientific Computing: An Introduction with Parallel Computing. Academic Press Professional, Inc.

Hore, A., Ziou, D., 2010. Image Quality Metrics: PSNR vs. SSIM, in: 2010 20th International Conference on Pattern Recognition, IEEE. pp. 2366-2369.

275 Joseph, P., 1982. An improved algorithm for reprojecting rays through pixel images. IEEE Transactions on Medical Imaging 1, 192-196.

Kak, A.C., Slaney, M., 2001. Principles of Computerized Tomographic Imaging. Society for Industrial and Applied Mathematics.

Paige, C.C., Saunders, M.A., 1982. LSQR: An algorithm for sparse linear equations and sparse least squares. ACM Transactions on Mathematical Software 8, 43-71.

Parcero, E., Flores, L., Sánchez, M., Vidal, V., Verdú, G., 2017. Impact of view reduction in CT on radiation dose for patients. Radiation Physics and Chemistry 137, 173-175.

Rahib, L., Smith, B.D., Aizenberg, R., Rosenzweig, A.B., Fleshman, J.M., Matrisian, L.M., 2014. Projecting Cancer Incidence and Deaths to 2030: The Unexpected Burden of Thyroid, Liver, and Pancreas Cancers in the United States. Cancer Research 74, 2913-2921.

Rodríguez-Alvarez, M.J., Sanchez, F., Soriano, A., Moliner, L., Sanchez, S., Benlloch, J.M., 2018. QR-factorization Algorithm for Computed Tomography (CT): Comparison with FDK and Conjugate Gradient (CG) Algorithms. IEEE Transactions on Radiation and Plasma Medical Sciences 2, 459-469.

Tang, X., Hsieh, J., Nilsen, R.A., Dutta, S., Samsonov, D., Hagiwara, A., 2006. A three-dimensional-weighted cone beam filtered backprojection (CB-FBP) algorithm for image reconstruction in volumetric CT-helical scanning. Physics in Medicine \& Biology 51, 855 .

Yan, K., Wang, X., Lu, L., Summers, R.M., 2018. Deeplesion: automated mining of large-scale lesion annotations and universal lesion detection with deep learning. Journal of Medical Imaging 5, 036501.

$\mathrm{Yu}, \mathrm{H}$., Wang, G., 2010. A soft-threshold filtering approach for reconstruction from a limited number of projections. Physics in Medicine and Biology 55, 3905.

Yu, W., Zeng, L., 2014. A Novel Weighted Total Difference Based Image Reconstruction Algorithm for Few-View Computed Tomography. PLoS ONE 9. 